\section{Unique clinico-biological, genetic and prognostic features of adult early T-cell precursor acute lymphoblastic leukemia}

Early T-cell precursor (ETP) acute lymphoblastic leukemia (ALL), was first identified within cases of childhood T-ALL based on its unique immunophenotypic and genetic features of limited (early) T-cell differentiation associated with (some) myeloid and stem cell features. ${ }^{1}$ Thus ETP-ALL blasts express CD7, dim CD5 ( $<75 \%$ positive cells), in the absence of CD1a and CD8, and positivity for $\geq 1$ myeloid/stem cell related markers (i.e., CD34, CD13 or CD33). ${ }^{1,2}$ In turn, ETP-ALL frequently shows myeloid-associated gene alterations such as FLT3, NRAS/KRAS, DNMT3A, IDH1 and IDH2 mutations, with lower frequencies of other T-ALL-associated mutations (e.g., NOTCH1 and CDKN2A/B gene mutations)., The World Health Organization (WHO) 2016 classification of ALL included ETP-ALL for the first time, as a provisional entity, ${ }^{7}$ but it failed to establish robust diagnostic criteria. Thus, after the first immunophenotypic characterization of ETP-ALL by Coustan-Smith et al. ${ }^{1}$ the proposed criteria did not allow identification of all ETP-ALL cases as detected by gene expression profiling. ${ }^{2}$ In addition, the "partial CD5 expression" criterion had a negative impact on the reproducibility of ETP-ALL diagnoses because of the lack of standardization of the method used for its assessment. Because of this, Zuubier et al. proposed refined immunophenotypic criteria by excluding CD5 expression while adding negativity for CD4. ${ }^{2}$

From the clinical point of view early studies based on limited numbers of pediatric patients indicated that ETPALL was associated with a very poor outcome. ${ }^{1,8,9}$ More recent data, based on larger series of children treated with more intensive therapy, showed no significant differences in outcome for ETP-ALL vs. other T-ALL cases. ${ }^{10}$ In contrast, limited data have been reported for adult ETP-ALL, with conflicting results. ${ }^{11,12}$ In one study, adult ETP-ALL was associated with a worse prognosis following different frontline chemotherapy schedules. ${ }^{11}$ The
GRAALL 2003 and 2005 trials showed that treatment intensification with allogeneic hematopoietic stem cell transplantation (HSCT) in ETP-ALL with early treatment resistance could improve survival. ${ }^{12}$

Here we retrospectively analyzed the frequency, clinico-biological and prognostic features of adult ETP-ALL vs. other T-ALL cases in a series of 185 adults with T-ALL treated within two consecutive minimal residual diseaseoriented trials by the Programa Español de Tratamientos en Hematología (PETHEMA): ALL-HR-2003 (NCT00853008) and ALL-HR-11 (NCT01540812), the latter still ongoing).

All 185 patients were diagnosed with T-ALL using the WHO criteria. The treatment protocol schedules have been described previously elsewhere. ${ }^{13,14}$ The patients' characteristics at diagnosis and at follow-up are summarized in Table 1. The immunological T-ALL subtype was defined according to the European Group for the Immunological Characterization of Leukemias (EGIL) criteria after centralized review of immunophenotypic reports. In turn, the criteria proposed by Zuurbier et al. ${ }^{2}$ were used to define ETP-ALL. The combination of markers used $(<5 \%$ CD1a, CD 8 and CD $4 ;>25 \%$ CD34 and/or CD33/CD13), resembles that proposed by CoustanSmith et al., while dim CD5 expression ( $<75 \%$ positive cells) was not considered. The inclusion of negativity for CD4 instead of dim CD5 expression as an immunophenotypic criterion, allows identification of most ETP-ALL cases identified by gene expression profiling (increased sensitivity of immunophenotyping to detect ETP-ALL cases). ${ }^{2}$ Clinical data were obtained in accordance with the principles of the Declaration of Helsinki and Spanish legislation, after written informed consent had been provided by each patient. The study was approved by the Institutional Review Board of the Hospital Germans Trias i Pujol (Badalona, Spain).

Comparisons between ETP-ALL and other T-ALL cases were performed with the $\chi^{2}$ test, Fisher exact test, and the median test, as appropriate. Event-free survival and overall survival curves were plotted using the Kaplan-Meier method and compared by the log-rank test. A secondary
A OS censored at HSCT

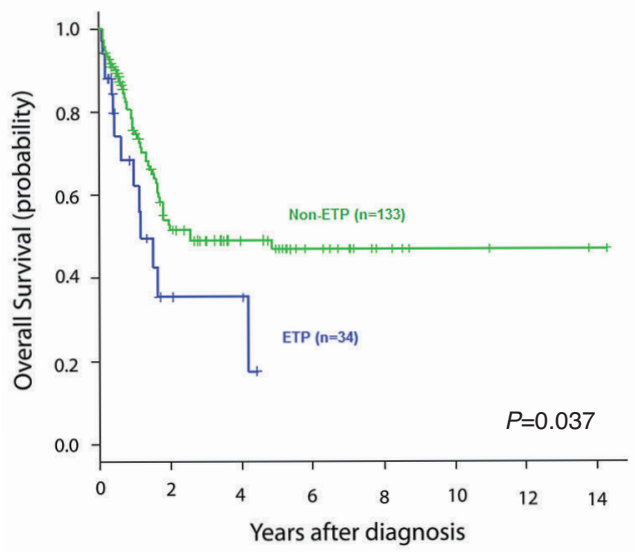

B OS non-censored at HSCT

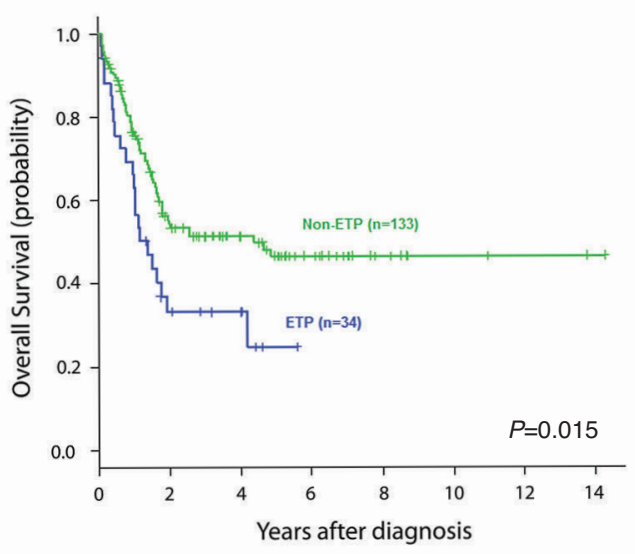

Figure 1. Impact of allogeneic hematopoietic stem cell transplantation on overall survival of patients with or without early T-cell precursor acute lymphoblastic leukemia. (A) Overall survival of adults with early T-cell precursor acute lymphoblastic leukemia (ETP-ALL) vs. other non-ETP T-ALL adult patients censored at transplantation; the survival probability, with 95\% confidence interval (95\% Cl) at 4 years was 36\% (95\% Cl: 13-59\%) for the ETP-ALL group and 49\% (95\% Cl: $39-59 \%)$ for the other non-ETP patients. (B) Overall survival of ETP and non-ETP T-ALL patients not censored at transplantation. The survival probability at 4 years was 33\% (95\% Cl: 16-50\%) for ETP-ALL patients vs. 51\% (95\% Cl: 42-60\%) for the non-ETP T-ALL group. 
Table 1. Clinico-biological and prognostic characteristics together with response to treatment of early T-cell precursor acute lymphoblastic leukemia vs. other types of T-cell acute lymphoblastic leukemia.

\begin{tabular}{|c|c|c|c|c|}
\hline & $\begin{array}{l}\text { Wholes series } \\
\quad(n=185)\end{array}$ & $\begin{array}{l}\text { EIP-ALL } \\
(n=34)\end{array}$ & $\begin{array}{l}\text { Other non-ETP T-ALL } \\
\qquad(n=133)\end{array}$ & $\mathbf{P}$ \\
\hline Age (years)* & $33.5(12)$ & $39(12)$ & $33(12)$ & 0.01 \\
\hline Female/male ratio & $49 / 136$ & $13 / 21$ & $30 / 103$ & 0.06 \\
\hline Lymph node enlargement & 65/163 (40\%) & 23/29 (79\%) & 66/118 (56\%) & 0.02 \\
\hline Splenomegaly & $61 / 178(34 \%)$ & $8 / 31(26 \%)$ & 49/130 (38\%) & 0.21 \\
\hline Hepatomegaly & 40/175 (23\%) & $7 / 31(23 \%)$ & $30 / 127(24 \%)$ & 0.90 \\
\hline Mediastinal mass & 86/182 (47\%) & $15(44 \%)$ & 65/131 (50\%) & 0.57 \\
\hline WBC count $\left(x 10^{9} / \mathrm{L}\right)^{*}$ & $94.3(121.0)$ & $72.2(155.1)$ & 96.6 (111.7) & 0.004 \\
\hline CNS involvement & 18/178 (10\%) & 6/33 (18\%) & $12 / 130(9 \%)$ & 0.21 \\
\hline Complex karyotype** & $9 / 141(6 \%)$ & $3 / 22(14 \%)$ & 6/104 (6\%) & 0.19 \\
\hline $\operatorname{Del}(6 q)$ & $10 / 140(7 \%)$ & $1 / 21(5 \%)$ & $9 / 104(9 \%)$ & 0.47 \\
\hline CDKN2A/B gene deletion & $31 / 53(58 \%)$ & $1 / 11(9 \%)$ & $29 / 40(73 \%)$ & $<0.001$ \\
\hline$T C R G$ gene deletion & 24/31 (77\%) & $3 / 8(37 \%)$ & 19/20 (95\%) & 0.003 \\
\hline Slow response at day +14 & 82/172 (48\%) & $27 / 33(82 \%)$ & 46/123 (37\%) & $<0.001$ \\
\hline 1 induction cycle to $\mathrm{CR}$ & 153/167 (92\%) & 19/26 (73\%) & 118/125 (94\%) & 0.003 \\
\hline 2 induction cycles to CR & $14 / 167(8 \%)$ & $7 / 26(27 \%)$ & $7 / 125(6 \%)$ & \\
\hline $\mathrm{CR}$ & $167 / 185(90 \%)$ & $26(77 \%)$ & 125/133 (94\%) & 0.005 \\
\hline MRD day $+35<0.1 \%$ & 106/141 (75\%) & $7 / 20(35 \%)$ & $90 / 110(82 \%)$ & $<0.001$ \\
\hline MRD day $+35<0.01 \%$ & 74/132 (44\%) & $3 / 20(15 \%)$ & 65/104 (63\%) & $<0.001$ \\
\hline Chemotherapy & 86/121 (71\%) & $6 / 20(30 \%)$ & 72/91 (79\%) & $<0.001$ \\
\hline Allogeneic HSCT & 35/121 (29\%) & $14 / 20(70 \%)$ & 19/91 (21\%) & \\
\hline
\end{tabular}

analysis of overall survival was performed, with transplanted patients censored at the time of allogeneic HSCT. Cumulative incidence of relapse and non-relapse-related mortality were analyzed as competing events with the Gray test. A Cox proportional hazard regression model was used to identify predictive factors for event-free and overall survival, whereas multivariate analysis for cumulative incidence of relapse was performed with the Fine and Gray model. Statistical significance was set at $P$-values $<0.05$.

Full immunophenotypic data according to the EGIL criteria were available for $167 / 185$ T-ALL patients; $34 / 167$ $(20 \%)$ T-ALL cases met criteria for ETP-ALL as defined by both Zuurbier et al. and the WHO. ${ }^{7}$ This frequency is similar to that observed in previously published cohorts of adults with T-ALL ${ }^{11,12}$ in which the phenotypic criteria applied in early pediatric studies to define ETP-ALL were used. ${ }^{1}$ Significant differences were observed when comparing ETP-ALL and non-ETP-ALL cases at diagnosis (Table 1). Patients with ETP traits were older [mean \pm standard deviation (SD): $39 \pm 12$ years vs. $33 \pm 12$ years, $P=0.01]$, more frequently had lymph node enlargement (79\% vs. $56 \%, P=0.02$ ), had lower white blood cell counts (mean \pm SD: $72.2 \pm 155.1 \times 10^{9} / \mathrm{L}$ vs. $96.6 \pm 111.7$ $\left.\mathrm{x} 10^{9} / \mathrm{L}, P=0.004\right)$, and showed a slightly different female to male ratio $(13 / 21$ vs. $30 / 103, P=0.06)$, similarly to what has been shown for the GRAALL cohort. ${ }^{12}$ In addition, our ETP-ALL cases frequently showed absence of $C D K N 2 A / B(P<0.001)$ and TCRG $(P=0.003)$ gene deletions. These results are in line with those previously reported in the literature ${ }^{6,14,16}$ and support the use of both genetic markers for the diagnosis of ETP-ALL. However, some discordance between immunophenotypic and genetic results was observed, which might be due to the limited sensitivity of current immunophenotypic criteria, highlighting the need for more precise and robust criteria and standardized methods for the diagnosis of ETP-ALL.

No significant differences in complete response, overall survival, event-free survival and cumulative incidence of relapse rates were observed between ETP-ALL patients enrolled in the two treatment protocols (data not shown). This allowed a combined evaluation of the data from both PETHEMA trials. ETP-ALL patients showed significantly poorer response to induction therapy compared to other T-ALL patients (Table 1). Thus, ETP-ALL patients had poorer bone marrow response $(<10 \%$ blasts) on day +14 compared to other T-ALL cases $(18 \%$ vs. $63 \%$, $P<0.001)$; half of ETP-ALL cases required second induction treatment (vs. $10 \%$ of the remaining T-ALL cases, $P<0.001)$ and $23 \%$ of them did not attain a complete response (vs. only $6 \%$ of other T-ALL cases, $P=0.005$ ). Minimal residual disease data were available for $132 / 167$ patients who reached a complete resposne after one or two induction cycles. Two-thirds (65\%) of ETP-ALL cases showed minimal residual disease levels $\geq 0.1 \%$ ( $v s$. $18 \%$ of non-ETP-ALL cases, $P<0.001)$, the percentage increasing to $85 \%$ (vs. $37 \%, P<0.001)$ at a cut-off level of $0.01 \%$. Consequently, as indicated per protocol, more ETP-ALL patients underwent allogeneic HSCT (70\% vs. $21 \%, P<0.001)$. Despite treatment intensification with allogeneic HSCT, ETP-ALL patients did not attain a significant improvement in the probability of overall sur- 
vival (Figure 1A, B). In fact, differences in overall and event-free survival between ETP-ALL and other non-ETP T-ALL patients were mostly due to the greater rate of failure to induction therapy, with a minimal contribution of the higher (non-statistically significant) cumulative incidence of relapse observed among ETP-ALL patients vs. other T-ALL cases (Online Supplementary Figure S1). However, it should be noted that the overall survival rate of adult T-ALL patients from the PETHEMA cohort who underwent allogeneic HSCT was lower than that observed in other studies. ${ }^{12,13}$ Thus, the improvement of non-transplant-related mortality in transplanted patients might have a positive impact on survival of ETP-ALL patients. Multivariate analyses showed that ETP-ALL was an independent adverse predictor for both overall survival and event-free survival [hazard ratio of 2.14 (95\% confidence interval: $1.29-3.53$ ), $P=0.003$ and 1.91 (1.18-3.11), $P=0.009$, respectively] together with a high white blood cell count (Online Supplementary Table S1).

In summary, here we confirmed the previously reported frequency and clinico-biological features of adult ETPALL and showed that adult ETP-ALL patients should be considered as a group with a worse prognosis. The poorer outcome of ETP-ALL patients in our study was due to a lower response to induction therapy and not to an increased relapse rate, which highlights the need to investigate the potential utility of alternative induction therapy for these patients. The use of acute myeloid leukemia-oriented chemotherapy, together with intensification of chemotherapy regimens, has been effective in clearing disease in pediatric ETP-ALL cases refractory to initial induction chemotherapy. ${ }^{17} \mathrm{~A}$ similar strategy could be applied to adult ETP-ALL patients. Based on these findings, and given the poor response attained in adults with the conventional induction regimen, it makes sense to explore the potential benefit of the FLAG-IDA regimen (idarubicin; fludarabine; ARA-C and granulocyte colonystimulating factor), a more myeloid-oriented chemotherapy schedule, as front-line induction treatment together with allogeneic HSCT after standard consolidation. In addition, new therapies targeting specific genes altered in ETP-ALL patients, such as FLT3 inhibitors, have emerged as novel alternative treatment strategies. However, the potential benefit of this latter treatment is restricted to ETP-ALL patients carrying FLT3 mutations in the tyrosine kinase domain (1/9, ETP-ALL cases in our cohort; data not shown). Similarly, other potential targeted therapies (e.g. Jak2 inhibitors or Bcl-2 antagonists) may also provide a benefit, but require further investigation.

Eulàlia Genescà, ${ }^{1 *}$ Mireia Morgades, ${ }^{2}$ Pau Montesinos, ${ }^{3}$ Pere Barba, ${ }^{4}$ Cristina Gil, ${ }^{5}$ Ramon Guàrdia, María-José Moreno, ${ }^{7}$ Daniel Martínez-Carballeira, ${ }^{8}$ Irene García-Cadenas," Susana Vives, ${ }^{2}$ Jordi Ribera, ${ }^{1}$ José González-Campos, ${ }^{10}$ Celia González-Gil, Lurdes Zamora, José-Luís Ramírez, Marina Díaz-Beya," Santiago Mercadal, ${ }^{12}$ María-Teresa Artola, ${ }^{13}$ Antònia Cladera, ${ }^{14}$ Mar Tormo, ${ }^{15}$ Arancha Bermúdez, ${ }^{16}$ Ferran Vall-Llovera, ${ }^{17}$ Pilar Martínez, ${ }^{18}$ María-Luz Amigo, ${ }^{19}$ Silvia Monsalvo, ${ }^{20}$ Andrés Novo, ${ }^{21}$ Marta Cervera, ${ }^{22}$ Antoni García-Guiñon, ${ }^{23}$ Jordi Juncà, ${ }^{2}$ Juana Ciudad, ${ }^{24}$ Alberto Orfao ${ }^{24}$ and Josep-Maria Ribera ${ }^{1,2}$

Josep Carreras Leukaemia Research Institute, Campus ICOGermans Trias i Pujol, Universitat Autònoma de Barcelona, Badalona; ${ }^{2}$ Clinical Hematology Department, ICO-Hospital Germans Trias $i$ Pujol, Universitat Autònoma de Barcelona, Badalona; ${ }^{3}$ Hospital Universitari i Politècnic La Fe \& CIBERONC, Instituto Carlos III
Madrid; ${ }^{4}$ Clinical Hematology Service, Hospital Universitari de la Vall d'Hebron, Barcelona; 'Clinical Hematology Service, Hospital General de Alicante, Alicante; ${ }^{6}$ Clinical Hematology Service, Hospital Josep Trueta-ICO, Girona; ${ }^{7}$ Clinical Hematology Service, Hospital Virgen de la Victoria, Málaga; ${ }^{8}$ Clinical Hematology Service, Hospital Universitario Central de Asturias, Oviedo; ${ }^{9}$ Clinical Hematology Service, Hospital de la Santa Creu i Sant Pau, Barcelona; ${ }^{10}$ Clinical Hematology Service, Hospital Virgen del Rocio, Sevilla; ${ }^{11}$ Clinical Hematology Service, Hospital Clinic de Barcelona, Barcelona; ${ }^{12}$ Clinical Hematology Service, Hospital Duran i Reynals-ICO, Hospitalet del Llobregat; ${ }^{13}$ Clinical Hematology Service, Hospital Universitario de Donostia, Donostia; ${ }^{14}$ Clinical Hematology Service, Hospital Son Llàtzer, Palma de Mallorca; ${ }^{15}$ Clinical Hematology Service, Hospital Clinico de Valencia, Valencia; ${ }^{16}$ Clinical Hematology Service, Hospital Marqués de Valdecilla, Santander; ${ }^{17}$ Clinical Hematology Service, Hospital Mútua de Terrassa, Terrassa; ${ }^{18}$ Clinical Hematology Service, Hospital 12 de Octubre, Madrid; ${ }^{19}$ Clinical Hematology Service, Hospital Morales Meseguer, Murcia; ${ }^{20}$ Clinical Hematology Service, Hospital Gregorio Marañón, Madrid; ${ }^{21}$ Clinical Hematology Service, Hospital Son Espases, Palma de Mallorca; ${ }^{22}$ Clinical Hematology Service, Hospital Joan XXIII, Tarragona; ${ }^{23}$ Clinical Hematology Service, Hospital Arnau de Vilanova, Lleida and ${ }^{24}$ Centro de Investigación del Cáncer (IBMCC-CSIC/USAL), Hospital Clínico Universitario de Salamanca, Instituto Bio-Sanitario de Salamanca, CIBERONC Salamanca, Spain

Acknowledgments: we would like to thank Rosa Coll, Rodrigo Martino, Raimundo García Boyero, Jesús Ma Hernández Rivas, Pilar Herrera, Juan Bergua, $M^{a}$ Jesús Peñarrubia, Eloy del Potro, Magdalena Sánchez, Beatriz Soria, Aurelio López Martinez, $M^{a}$ José Sánchez Sánchez, Pilar Bravo, Pilar Vivancos, Alfons Serrano, José Santiago Bermón, Maria Lourdes Amador Barciela and Matxalen Olivares Salaverri for providing retrospective clinical data for this study.

Funding: this project was supported by the Asociación Española Contra el Cáncer (project ref: GC16173697BIGA), PI14/01971 FIS, Instituto Carlos III, CERCA Program/Generalitat de Catalunya, 2014-SGR225 (GRE), Obra Social "La Caixa". This work was also partially supported by FEDER funds from the ISCIII (PT13/0010/0026, CIBERONC (CB16/12/00284 and CB16/12/00400), Madrid, Spain). P. Barba was supported by the Instituto de Salud Carlos III FIS16101433 and PERIS 2018-2020 from Generalitat de Catalunya (BDNS357800) grants.

Correspondence:

EULALIA GENESCA' - egenesca@carrerasresearch.org

doi:10.3324/haematol.2019.225078

Information on authorship, contributions, and financial $\&$ other disclosures was provided by the authors and is available with the online version of this article at www. haematologica.org.

\section{References}

1. Coustan-Smith E, Mullighan CG, Onciu M, et al. Early T-cell precursor leukaemia: a subtype of very high-risk acute lymphoblastic leukaemia. Lancet Oncol. 2009;10(2):147-156.

2. Zuurbier L, Gutierrez A, Mullighan CG, et al. Immature MEF2C-dysregulated $\mathrm{T}$-cell leukemia patients have an early $\mathrm{T}$-cell precursor acute lymphoblastic leukemia gene signature and typically have nonrearranged T-cell receptors. Haematologica. 2014;99(1):94-102.

3. Neumann M, Heesch S, Gokbuget N, et al. Clinical and molecular characterization of early $\mathrm{T}$-cell precursor leukemia: a high-risk subgroup in adult T-ALL with a high frequency of FLT3 mutations. Blood Cancer J. 2012;2(1):e55.

4. Neumann M, Coskun E, Fransecky L, et al. FLT3 mutations in early T-cell precursor ALL characterize a stem cell like leukemia and imply the clinical use of tyrosine kinase inhibitors. PLoS One. 2013;8(1):e53190

5. Zhang J, Ding L, Holmfeldt L, et al. The genetic basis of early T-cell precursor acute lymphoblastic leukaemia. Nature. 2012;481(7380):157-163.

6. Van Vlierberghe P, Ambesi-Impiombato A, De Keersmaecker K, et al. 
Prognostic relevance of integrated genetic profiling in adult T-cell acute lymphoblastic leukemia. Blood. 2013;122(1):74-82.

7. Arber DA, Orazi A, Hasserjian R, et al. The 2016 revision to the World Health Organization classification of myeloid neoplasms and acute leukemia. Blood. 2016;127(20):2391-2405.

8. Inukai T, Kiyokawa N, Campana D, et al. Clinical significance of early T-cell precursor acute lymphoblastic leukaemia: results of the Tokyo Children's Cancer Study Group Study L99-15. Br J Haematol. 2012;156(3):358-365.

9. Ma M, Wang X, Tang J, et al. Early T-cell precursor leukemia: a subtype of high risk childhood acute lymphoblastic leukemia. Front Med. 2012;6(4):416-420.

10. Patrick K, Wade R, Goulden N, et al. Outcome for children and young people with Early T-cell precursor acute lymphoblastic leukaemia treated on a contemporary protocol, UKALL 2003. Br J Haematol. 2014;166(3):421-424.

11. Jain N, Lamb AV, O'Brien S, et al. Early T-cell precursor acute lymphoblastic leukemia/lymphoma (ETP-ALL/LBL) in adolescents and adults: a high-risk subtype. Blood. 2016;127(15):1863-1869.

12. Bond J, Graux C, Lhermitte L, et al. Early response-based therapy stratification improves survival in adult early thymic precursor acute lymphoblastic leukemia: a Group for Research on Adult Acute
Lymphoblastic Leukemia study. J Clin Oncol. 2017;35(23):26832691.

13. Barba P, Morgades M, Montesinos $\mathrm{P}$, et al. Increased survival due to lower toxicity for high risk T-cell acute lymphoblastic leukemia patients in 2 consecutive pediatric-inspired PETHEMA trials. Eur J Haematol. 2018;102(1):79-86.

14. Genesca E, Lazarenkov A, Morgades M, et al. Frequency and clinical impact of CDKN2A/ARF/CDKN2B gene deletions as assessed by indepth genetic analyses in adult $\mathrm{T}$ cell acute lymphoblastic leukemia. J Hematol Oncol. 2018;11(1):96.

15. Gutierrez A, Dahlberg SE, Neuberg DS, et al. Absence of biallelic TCRgamma deletion predicts early treatment failure in pediatric Tcell acute lymphoblastic leukemia. J Clin Oncol. 2010;28(24):38163823.

16. Vicente C, Schwab C, Broux M, et al. Targeted sequencing identifies associations between IL7R-JAK mutations and epigenetic modulators in T-cell acute lymphoblastic leukemia. Haematologica. 2015;100(10):1301-1310.

17. Conter V, Valsecchi MG, Buldini B, et al. Early T-cell precursor acute lymphoblastic leukaemia in children treated in AIEOP centres with AIEOP-BFM protocols: a retrospective analysis. Lancet Haematol. 2016;3(2):e80-86 